\title{
On the mechanisms of natural transport in the solar system
}

\author{
Yuan Ren* Josep J. Masdemont* Gerard Gómez** \\ Elena Fantino* \\ * IEEC \& Departament de Matemàtica Aplicada I, ETSEIB, \\ Universitat Politècnica de Catalunya, Diagonal 647, 08028 Barcelona, \\ Spain(email: yuan.ren@upc.edu). \\ ** IEEC \& Departament de Matemàtica Aplicada i Anàlisi, Universitat \\ de Barcelona, Gran Via 585, 08007 Barcelona, Spain (e-mail: \\ gerard@maia.ub.es)
}

\begin{abstract}
:
Some minor bodies in the solar system (i.e., comets, asteroids and planetary ejecta) are capable of performing transfers from their original location to very distant places, provided they possess sufficiently large energies. Some of them can reach the surface of a planet. This phenomenon is called natural transport. Within the planar circular restricted three-body problem (PCR3BP) with the Sun and a planet as primaries, and also within two coupled PCR3BPs, the gravity of the secondary causes long-term perturbations and the minor body performs swingbys at the secondary, thus resulting in different behaviours with respect to those observed in the heliocentric two-body model. In this contribution, two natural transport mechanisms in the PCR3BP framework are considered. The first is a short-time transport, consisting in heteroclinic connections between libration point orbits of pairs of Sun-planet PCR3BPs: by varying the relative orbital phase of the involved planets at the start of the transfer, the location of the Poincare section at which the connection is sought and the size of the departure and arrival periodic orbits, the intersection between the associated unstable and stable manifolds (respectively in the departure and arrival PCR3BP) is computed. The second mechanism corresponds to a long-time transport, the result of the strongly chaotic motion of the minor body in the PCR3BP: the heliocentric orbit changes significantly due to the gravitational interactions with the Sun and the planets (especially the giant planets), and this eventually allows the minor body to reach the vicinity of some planet. In this contribution we provide an analysis of the natural transport in solar system by these two mechanisms. In particular we discuss the key properties of the natural transport, such as the possibility of transferring between two specified celestial bodies, the type of transport and the time of flight. The final aim is to get a deeper insight into the motion of the minor bodies and the exchange of natural material in the solar system.
\end{abstract}

Keywords: Natural transport, Poincaré section, Three-body problem, Lobe dynamics.

\section{INTRODUCTION}

The natural transport is the phenomenon in which particles of natural material transfer from their original orbit to very distant locations in the solar system. It is considered a crucial issue in the material exchange between terrestrial planets. The PCR3BP is a chaotic system, as the motion of the third body is highly sensitive to initial conditions and parameter values. In this system, the natural transport shows very different behaviors from those observed in the

\footnotetext{
^ E.Fantino and Y.Ren have been supported by the Marie Curie Actions Research and Training Network AstroNet MCRTN-CT2006-035151. G. Gómez and J.J. Masdemont have been partially supported by the grants MTM2006-05849/Consolider, MTM200906973 and 2009SGR859. The authors also acknowledge the use of EIXAM, the UPC Applied Math cluster system for research computing (see http://www.ma1.upc.edu/eixam/), and in particular Pau Roldan for providing technical support in the use of the cluster.
}

two-body model due to the long-term perturbations caused by the second primary and the effect of close passages. The natural transport has been investigated by many authors, in the general framework as well as relative to specific cases: for the Earth-Moon transport the reader is referred to (Bollt and Meiss, 1995b,a; Meiss and Ott, 1986), for the Mars-Earth transport to (Gladman, 1997, 1996a,b), and in general to (Dellnitz et al., 2005). In this paper, two types of natural transport in the PCR3BP are investigated: the short-time natural transport within two coplanar coupled PCR3BPs, i.e., direct connections between the manifolds of a pair of PCR3BPs, and the long-time natural transport in the PCR3BP, based on the analysis of the chaotic motion in this model.

For a definition of the adopted dynamical models (i.e., the PCR3BP, and the coupled PCR3BPs) the reader is referred to (Szebehely, 1967; Parker and Chua, 1989; Gómez 
et al., 2001). The analysis of the short-time natural transport between planets in solar system is presented in Sect. 2 ), whereas in Sect. 3 the process to determine the longtime natural transport is described. The natural transport from Mars to the Earth is used as an example throughout the paper. However, the method can be extended to other pairs of planets or other three-body systems.

\section{SHORT-TIME NATURAL TRANSPORT}

The short-time natural transport is based on the existence of heteroclinic connections between libration point orbits (LPOs), and in particular Lyapunov orbits, of a pair of consecutive Sun-planet PC3BPs, i.e., such that the two involved planets are on consecutive orbits in the solar system. Initially moving on the unstable manifold of a LPO of the departure PC3BP, the third body passes onto the stable manifold of a LPO of the arrival PC3BP and eventually reaches the vicinity of the corresponding planet. The dynamical model switch happens in the region where the two given invariant manifolds intersect in phase space. The existence of such intersection is the necessary condition for the specific short-time transport to take place. Fig. 1 illustrates the outer branches of the stable and unstable manifolds of a Sun-Earth $L_{2}$ Lyapunov orbit and the inner branches of the stable and unstable manifolds of a SunMars $L_{1}$ Lyapunov orbit. For convenience, each invariant manifold has been drawn in the respective synodic reference frame, a simplification which in the present case does not affect the values of the distances of the two objects from the Sun. Fig. 1 shows that the two sets of objects stay well separated from each other. Computations extended over much longer times indicate that the intersection between a Sun-Mars and a Sun-Earth invariant manifold never occurs. The manifolds of other types of LPOs look similar. In this respect, they can be represented and replaced by the invariant manifolds of the corresponding libration points. Fig. 2 shows the invariant manifolds of the $L_{1}$ and $L_{2}$ points of a generic PCR3BP. Their minimum

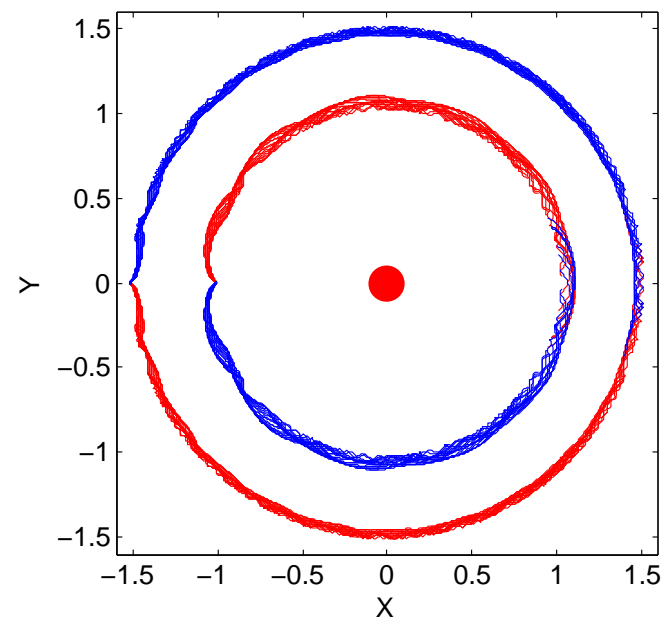

Fig. 1. Branches of stable (blue) and unstable (red) invariant manifolds, respectively of periodic orbits around $L_{1}$ in the Sun-Mars PCR3BP and $L_{2}$ in the Sun-Earth PCR3BP. This plot has been obtained by superimposing the respective synodic reference frames.
Table 1. The minimum and maximum heliocentric distances of the invariant manifolds of the $L_{1}$ and $L_{2}$ points of the Sun-planet PC3BPs. Quantities expressed in astronomical units. Asterisks indicate the consecutive Sunplanet systems which satisfy the necessary condition for the short-time transfer.

\begin{tabular}{|l|c|c|c|c|}
\hline \multirow{2}{*}{ PCR3BP } & $R\left[W_{L_{1}}^{u(s)}\right]$ & \multicolumn{2}{|c|}{$R\left[W_{L_{2}}^{u(s)}\right]$} \\
\cline { 2 - 5 } & $\min$ & $\max$ & $\min$ & $\max$ \\
\hline Sun-Mercury & 0.37547 & 0.38562 & 0.38858 & 0.39918 \\
\hline Sun-Venus & 0.67152 & 0.71658 & 0.73011 & 0.78024 \\
\hline Sun-Earth & 0.92328 & 0.98998 & 1.01008 & 1.08488 \\
\hline Sun-Mars & 1.46684 & 1.51644 & 1.53094 & 1.58331 \\
\hline Sun-Jupiter & 3.02493 & 4.85550 & 5.56589 & $9.46402^{*}$ \\
\hline Sun-Saturn & $6.63467^{*}$ & 9.12494 & 9.99818 & 14.04464 \\
\hline Sun-Uranus & 15.87518 & 18.75222 & 19.69233 & $23.49574^{*}$ \\
\hline Sun-Neptune & $23.35734^{*}$ & 29.33805 & 30.89615 & 37.25573 \\
\hline
\end{tabular}

and maximum distances from the center of mass of the system are labelled $R\left[W_{L_{1}}^{u}\right]_{\min }$ and $R\left[W_{L_{2}}^{u}\right]_{\max }$, respectively. When referred to a Sun-planet PCR3BP, such quantities constitute a good approximation of the minimum and maximum distances from the Sun, given that the mass ratio of any Sun-planet system is always a very small number. Table 1 reports the values of these quantities for all the Sun-planet PCr3BPs (except Sun-Pluto). The necessary condition for the short-time transfer can then be translated into the following inequality:

$$
R_{\max }\left[W_{L_{2}}^{u(s)}\right]_{\text {inner }} \geq R_{\min }\left[W_{L_{1}}^{u(s)}\right]_{\text {outer }} .
$$

It states that the short-time transport between consecutive Sun-planet systems is possible only if the maximum heliocentric distance of the inner problem is larger or equal to the minimum heliocentric distance of the outer problem, thus expressing in a quantitative way the intersection condition. According to Table 1, only two short-time connections are possible in the solar system, i.e., Jupiter-Saturn

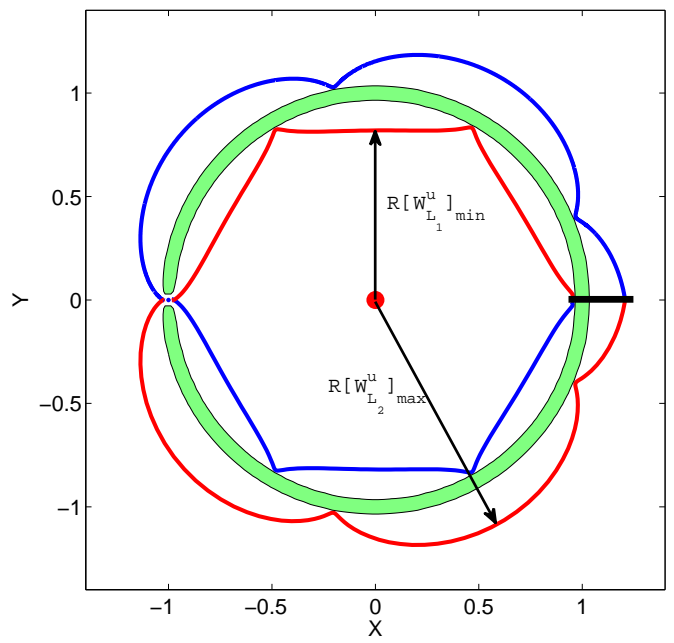

Fig. 2. Branches of the stable and unstable invariant manifolds of the $L_{1}$ and $L_{2}$ libration points in a PCR3BP. The minimum and maximum distances from the center of mass of the system are labelled $R\left[W_{L_{1}}^{u}\right]_{\min }$ and $R\left[W_{L_{2}}^{u}\right]_{\max }$, respectively. 


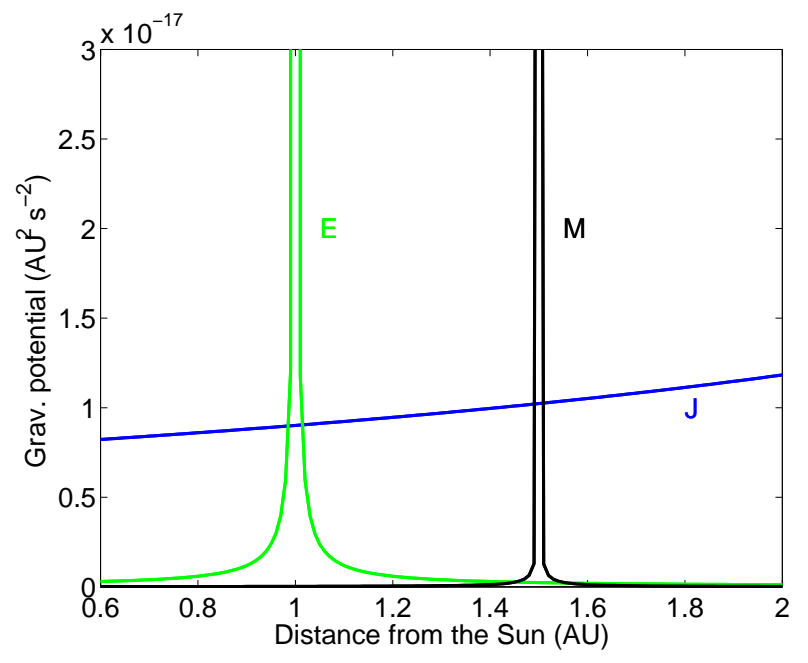

Fig. 3. The relative importance among the gravity of the Earth (green), Mars (black) and Jupiter (blue) in the heliocentric region including the orbits of the Earth and Mars.

and Uranus-Neptune (denoted by asterisks). We conclude that not only the short-time transport between Mars and the Earth is not possible, but that this is the most common situation in our planetary system.

\section{LONG-TIME NATURAL TRANSPORT}

The short-time transport concept cannot comprehensively explain the exchange of natural material throughout our solar system, and it completely fails in the case of the terrestrial planets. This imposes to evaluate alternative mechanisms. The scenario proposed in this contribution is the long-time transport based on the study of the chaoticity within a PCR3BP, and in particular the analysis of transport between fixed points on the Poincaré maps or lobe dynamics. The Mars-to-Earth transport is used as an example. Apart from the Sun, the gravity of the Earth, Mars and Jupiter are the dominant forces in the region between the orbits of Mars and the Earth (Fig. 3). For this reason, the long-time natural transport here discussed is based on the Sun-Jupiter PCR3BP. The chaoticity of the system is analyzed by considering Poincaré maps drawn at several energy levels. The adopted Poincaré section is the $x \dot{x}$ plane in the Sun-Jupiter PC3BP synodic barycentric reference frame, supplemented by the relations $\tan ^{-1}(y / x)=180^{\circ}$ and $\dot{y}<0$. In this way, a point on the Poincaré section corresponds to a well defined state, given that $y=0$, and $\dot{y}$ is straightforwardly determined by the previous conditions, once the Jacobi constant $C$ is given. By forward propagating a given initial state over a short time (i.e., $<2 \pi$ in Sun-Jupiter adimensional time units), a short trajectory segment in synodic coordinates is obtained, corresponding to an almost complete elliptical orbit in inertial space. By propagating a dense set of initial conditions for $C=3.03$, we have determined the perihelion and aphelion distances of the corresponding inertial orbits, based on which we have divided the Poincaré section into regions (Fig. 4): the two "forbidden" regions correspond to impossible motion at the given energy level; "region 1" contains initial states of inertial orbits that do not intersect the orbits of the Earth and Mars; the initial states in

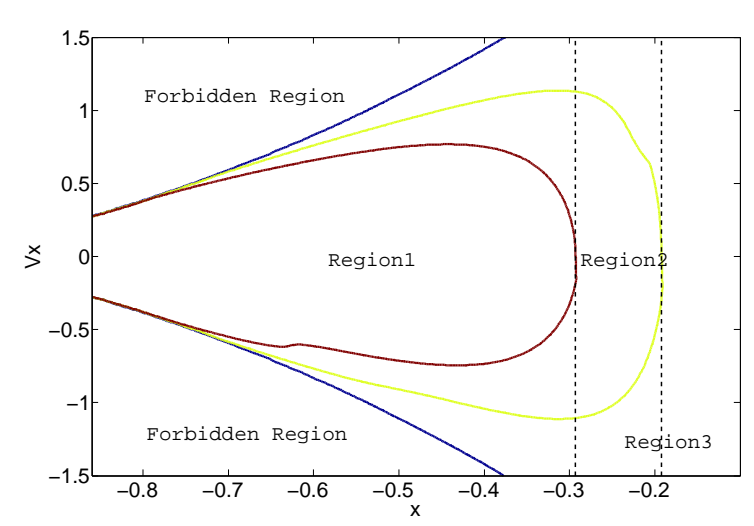

Fig. 4. Subdivision of the initial conditions distributed on the Poincaré section for $C=3.03$ into regions describing the extension (perihelion, aphelion) of the corresponding heliocentric inertial orbits relative to the orbits of the Earth and Mars.

"region 2" produce orbits which only intersect the orbit of Mars; finally, the initial states in "region 3" correspond to motions which have intersections with the orbit of the Earth. Therefore finding transport from "region 2" to "region 3" on the Poincaré section is equivalent to finding transport from Mars to the Earth in configuration space.

\subsection{Poincaré maps}

Fig. 5 shows four Poincaré maps for the Sun-Jupiter PCR3BP, characterized by decreasing value of $C$. Each of them has been obtained by setting a mesh grid on the Poincaré section, forward propagating the corresponding initial states and marking their successive intersections with the section. The four plots have been obtained from an initial mesh grid of 35 by 25 points such that $-0.86048 \leq x \leq-0.15$ and $-0.2 \leq \dot{x} \leq 0.2$, with $C$ $=3.14,3.09,3.06$ and 3.03, respectively. They illustrate the first $10^{3}$ returns. As such, they give an insight into the structure of the phase space at varying energy. In particular, it is possible to detect islands (periodic orbits) and fixed points at their centers. When transformed into configuration space, the periodic orbits correspond to invariant tori and the fixed points to periodic orbits. The only possible transfer between different regions of the Poincaré map can only happen through the gaps in the chain of islands, i.e. through the chaotic sea. A close look at the four plots of Fig. 5 shows that the size of the chaotic sea increases as the Jacobi constant decreases (i.e., the energy increases), and in particular when $C=3.03$ "region 2 " and a part of "region 3" are in the chaotic sea, thus suggesting that at this energy level the long-time natural transport from Mars to the Earth may be possible.

\subsection{Fixed points and their manifolds}

Fixed points can either be found at the center of the islands or in the chaotic sea, the latter case being associated to the transport. The most efficient way to identify the fixed points on the Poincaré map is by either the shooting method or the parallel shooting method, respectively when the order (periodicity, or number of distinct successive returns) of the fixed point is lower or greater than five, 

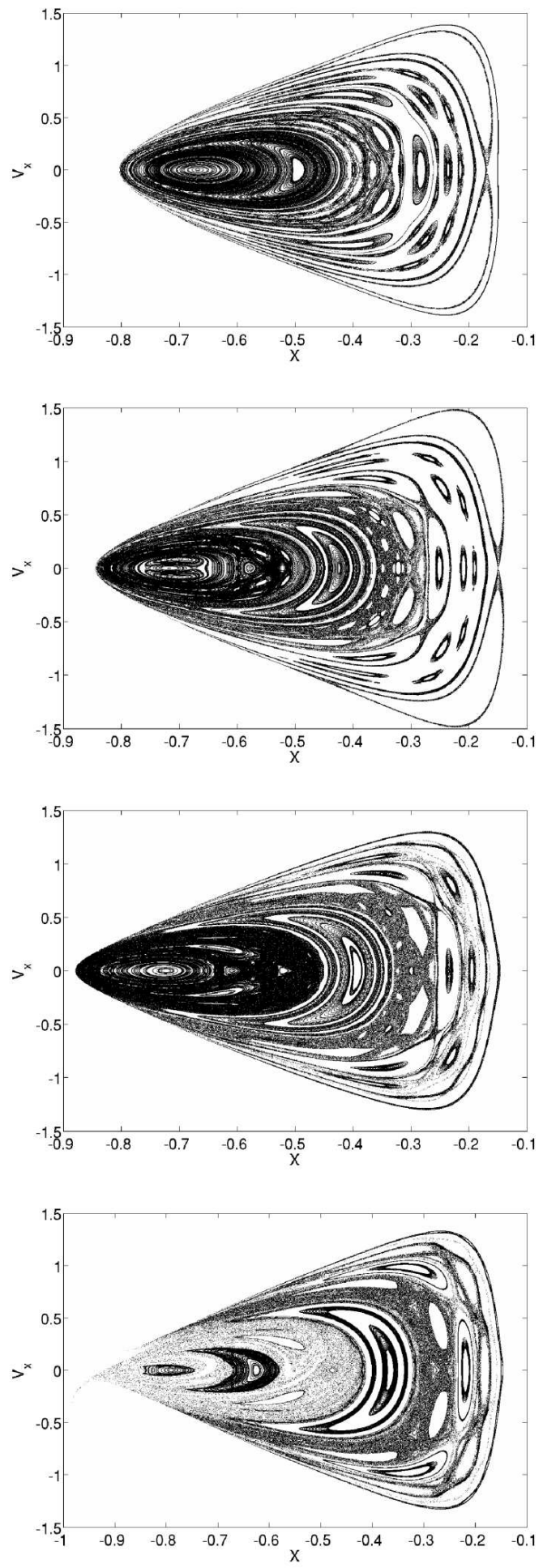

Fig. 5. Poincaré maps of the Sun-Jupiter PCR3BP corresponding to $C=3.14,3.09,3.06,3.03$, respectively from top to bottom.

the reason being the inability of the shooting method to converge to a high-order fixed point, with the tendency of detecting the nearest fixed point of order less than five.

The shooting method aims at zeroing the difference

$$
\mathbf{F}=\left[\begin{array}{c}
x \\
\dot{x}
\end{array}\right]_{t_{f}}-\left[\begin{array}{c}
x \\
\dot{x}
\end{array}\right]_{t_{0}}
$$

between the final state (i.e, after a chosen number of returns) and the initial state on the Poincaré section. By setting $\mathbf{x}=\left[\begin{array}{l}x \\ \dot{x}\end{array}\right]_{t_{0}}$, the variation of $\mathbf{F}$ can be expressed as

$$
\frac{d \mathbf{F}}{d \mathbf{x}}=(\mathbf{\Phi}-\mathbf{I}) \Delta \mathbf{x}
$$

where $\mathbf{I}$ is the $2 \times 2$ identity matrix. $\boldsymbol{\Phi}$ is a $2 \times 2$ matrix obtained as

$$
\boldsymbol{\Phi}_{2 \times 2}=\left[\begin{array}{lll}
\frac{\partial x_{f}}{\partial x_{0}} & \frac{\partial x_{f}}{\partial \dot{x}_{0}} & \frac{\partial x_{f}}{\partial \dot{y}_{0}} \\
\frac{\partial \dot{x}_{f}}{\partial x_{0}} & \frac{\partial \dot{x}_{f}}{\partial \dot{x}_{0}} & \frac{\partial \dot{x}_{f}}{\partial \dot{y}_{0}}
\end{array}\right]_{2 \times 3}\left[\begin{array}{cc}
1 & 0 \\
0 & 1 \\
\frac{\partial \dot{y}_{0}}{\partial x_{0}} & \frac{\partial \dot{y}_{0}}{\partial \dot{x}_{0}}
\end{array}\right]_{3 \times 2} .
$$

The $2 \times 3$ matrix in the above product represents the variation from the initial to the target Poincaré section. $\partial \dot{y}_{0} / \partial x_{0}$ and $\partial \dot{y}_{0} / \partial \dot{x}_{0}$ in the $3 \times 2$ matrix on the right account for the dependence of $\dot{y}_{0}$ on $x_{0}$ and $\dot{x}_{0}$ (through $\left.C\right)$. $\mathbf{F}$ is driven to zero by Newton-Raphson. In practice, the process is divided into two steps: a first search is conducted with a large tolerance because different initial guesses tend to converge to the same fixed point, and the use of a strict tolerance would only increase the computation time. Then, the distance between the final points is checked: whenever two points are closer than twice the tolerance, one of them is simply the duplicate of the other and is eliminated. A successive application of the shooting method with a stricter tolerance allows to refine the estimation of the fixed points that have survived the first search.

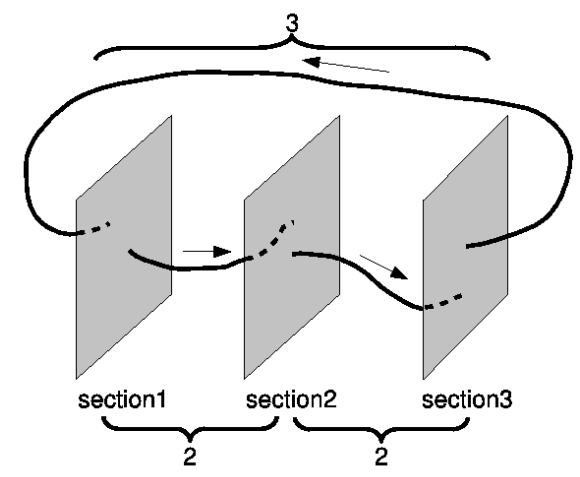

Fig. 6. Sketch of the parallel shooting method.

The parallel shooting method introduces additional, intermediate Poincaré sections, as illustrated in Fig. 6: here the order of the fixed point is 7 , and two intermediate sections are set (although for this order one section would be sufficient). Section 2 and section 3 represent respectively the second and the fourth crossing of the trajectory through the Poincaré section. The mesh grids are set on all the sections and the nodes on different sections are combined together to form a set of initial guesses. The side effect of this new method is a considerable increase in the computation time, but the strategy allows to detect fixed points to any order.

The stability of a fixed point depends on the modulus of the eigenvalues of its monodromy matrix. Since the monodromy matrix is a $2 \times 2$ matrix, it has two eigenvalues. A 
Table 2. Position $(x, \dot{x})$, stable and unstable eigenvalues ( $E^{s}$ and $E^{u}$ ) and period (in adimensional time units, $1 \mathrm{TU} \approx 12 / 2 \pi$ years $=1.9$ years) for the detected saddle points in the Sun-Jupiter PCR3BP.

\begin{tabular}{|c|c|c|c|c|c|}
\hline Order & 4 & 4 & & & 8 \\
\hline & & &
\end{tabular}

saddle point is a fixed point for which the two eigenvalues have modulus greater and smaller than unity, respectively. There are stable and unstable manifolds associated to a saddle point and the eigenvectors corresponding to the stable and unstable eigenvalues constitute the linear approximation of the stable and unstable manifolds, respectively.

Fig. 7 shows all the fixed points identified in the SunJupiter PCR3BP for $C=3.0$. All the fixed points in the center of the islands are stable, whereas the saddle points appear at the gaps of the island chain. Also the invariant manifolds of the fixed points are represented. There are five sets of saddle points. Their position on the Poincaré section, their eigenvalues and the period of the corresponding periodic orbit in configuration space are reported in Table 2: an $n$-periodic orbit is identified by $n$ fixed points, but for the sake of brevity, the table provides data only for one of them, the others being the result of iterating $n-1$ times.

\subsection{Lobe dynamics}

Fig. 7 shows that the manifolds of one of the order4 saddle points have intersections with the orbit of the Earth, the manifolds of the order-5 saddle point have intersections with the orbit of Mars, and these manifolds have intersections with each other. Thus, the natural

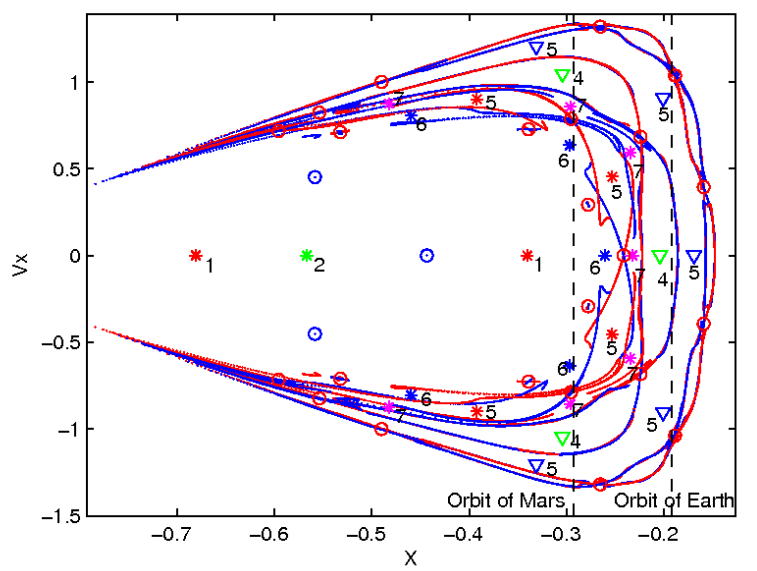

Fig. 7. Fixed points and their invariant manifolds in the Sun-Jupiter PCR3BP at $C=3.0$ : saddle points (o), stable fixed points $(\star$ and $\nabla$ ) and their invariant manifolds. transport can be realized with the aid of these manifolds: the particle leaves Mars following the unstable manifold of the order- 5 orbit, then switches to the stable manifold of the order-4 orbit at the intersection of these two manifolds, passes through the gap in the chain of islands, simultaneously switches to the unstable manifold of the order-4 orbit and intersects the orbit of the Earth. The region enclosed by the stable and unstable manifolds is called "lobe". The transport between regions of the phase space can be completely described by the dynamical evolution of the lobe. Hence, by choosing a set of initial states in the lobe, backward and forward propagation are performed until the transport from "region 2" to "region 3" (i.e., from the orbit of Mars to that of the Earth) is achieved. Fig. 8 illustrates an example of lobe evolution: $T$ represents the initial states near the intersection of the manifolds of the order- 4 and order- 5 saddle points. After 21 iterations in the forward direction, some of these states move from "region 2" to "region 3". However, since such states were initially already very close to "region 3 " (i.e., the perihelions of their initial orbits were very close to the orbit of the Earth), it means that the effect of the gravity of Jupiter is minor in this case.

By an additional backward propagation, an initial orbit with the perihelion far from the orbit of the Earth is sought, thus obtaining an overall transfer with a significant effect of the perturbations of Jupiter. Figure 9 shows the orbit of a long-time natural transport from Mars to the Earth found by this procedure. The inertial frame

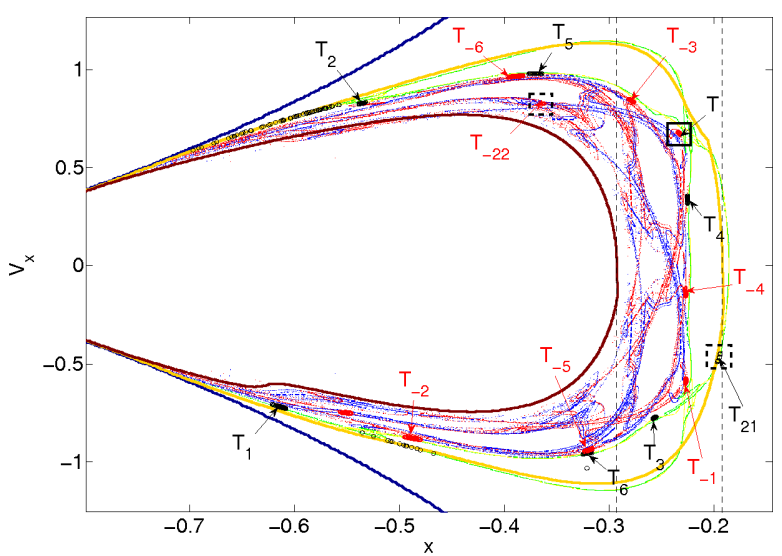

Fig. 8. Lobe evolution. 

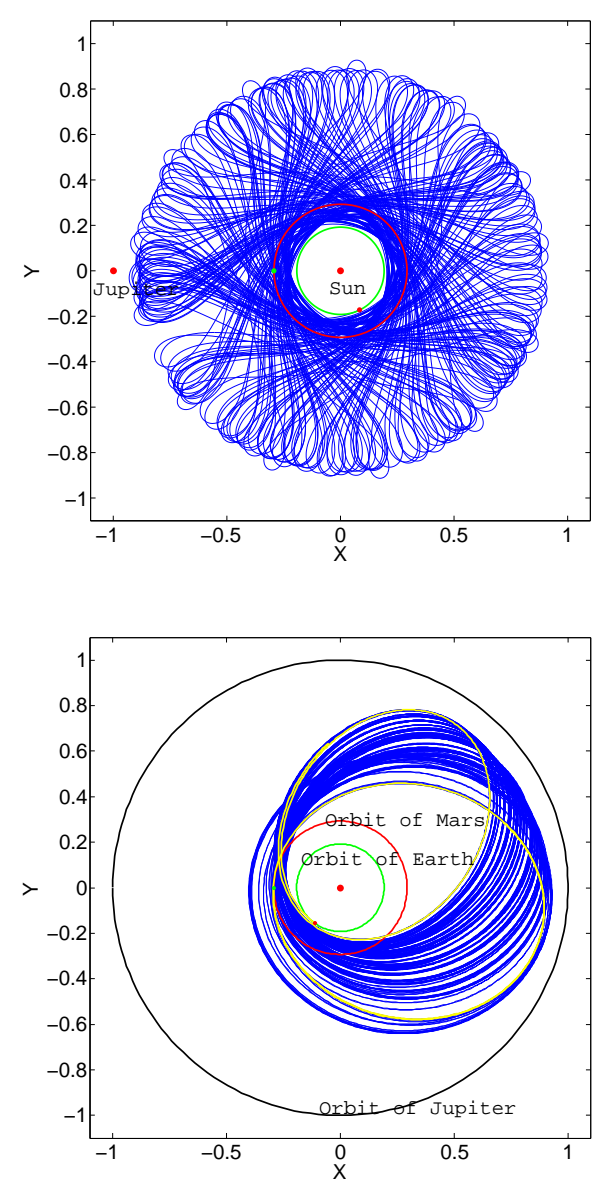

Fig. 9. The Mars-to-Earth transfer orbit corresponding to the long-time natural transport described in the text for $C=3.0$. Synodic barycentric reference frame view at the top, inertial heliocentric reference frame plot at the bottom.

view shows that the initial trajectory does not have any intersection with the orbit of the Earth. Then, as a result of the gravity of Jupiter, the orbit gradually evolves, eventually reaching the vicinity of the Earth's orbit. This specific transport involves 161 iterations corresponding to 671.32 time units in the Sun-Jupiter system, or 1282.6 years. We note, however, that the transfer orbit found by this method only guarantees a connection between the orbits of the Earth and Mars, not between the two planets. It certainly proves the possibility of the natural transport at this specific energy level, but the real transport from the surface of Mars to that of the Earth may require much longer times.

\section{CONCLUSIONS}

Two techniques for the analysis of the natural transport in the solar system have been presented. Each of them has a preferential framework of application. The first method achieves the short-time transport, with application to the design of spacecraft trajectories between Jupiter and Saturn and tours of Jupiter's icy moons. The second method is the long-time alternative for the cases in which the manifolds of the coupled PCR3BPs do not present direct intersection, such as the Mars-to-Earth case. Our numeri- cal simulations prove that the second method is capable of describing the transfer of the martian ejecta with aphelion near the orbit of Jupiter to the region of the Earth's orbit. Nevertheless, the most appropriate framework for the study of the low-energy, natural transport between, e.g., Mars and the Earth is the $n$-body model (see (Gladman, 1997, 1996b)), since the close passages at the Earth and Mars (not accounted for in the Sun-Jupiter PCR3BP) may be relevant. An attempt at describing the natural transport according to dynamical models of growing complexity (three-body, quasi-bicircular, $n$-body models) will be the subject of a subsequent publication.

\section{REFERENCES}

E. M. Bollt and J. D. Meiss. Targeting Chaotic Orbits to The Moon Through Recurrence. Physics Letters A, 204 (5/6):373-378, 1995a.

E. M. Bollt and J. D. Meiss. Controlling Chaotic Transport Through Recurrence. Physica D: Nonlinear Phenomena, 81(3):280-294, 1995b.

M. Dellnitz, O. Junge, W. S. Koon, F. Lekien, M. L. Lo, J. E. Marsden, K. Padberg, R. Preis, S. D. Ross, and B. Thiere. Transport in Dynamical Astronomy and Multibody Problem. International Journal of Bifurcation and Chaos, 15(3):699-727, 2005.

B. Gladman. The Exchange of Impact Ejecta Between Terrestrial Planets. Science, 217:1387-1392, 1996a.

B. Gladman. Destination Earth: Martian meteorite delivery. Icarus, 130:228-246, 1997.

B. Gladman. Delivery of Planetary Ejecta to Earth. PhD thesis, Cornell University, August 1996b.

G. Gómez, A. Jorba, J. Llibre, R. Martinez, J. Masdemont, and C. Simó. Dynamics and Mission Design near Libration Points, Vol. I-IV. World Scientific Publishing Co., Singapore, 2001.

J. D. Meiss and E. Ott. Markov Tree Model of Transport in Area-preserving Maps. Physica D: Nonlinear Phenomena, 20(2/3):387-402, 1986.

T.S. Parker and L.O. Chua. Practical Numerical Algorithms for Chaotic Systems. Springer-Verlag, Berlin, 1989.

V. Szebehely. Theory of Orbits in the Restricted Problem of Three Bodies. Academic Press, New York, 1967. 